\title{
Two Novel Variants in NLRP7 Gene in an Egyptian Female Patient with Consecutive Molar Pregnancies Complicated by Choriocarcinoma
}

\author{
Taghreed A Shalabi ${ }^{1}$, Mohamed S Abdel-Hamid ${ }^{2}$, Mai M Shaker ${ }^{3}$
}

\begin{abstract}
Background: Hydatidiform mole, whether complete or partial mole, is one of the most common forms of gestational trophoblastic disease. It is characterized by extreme trophoblastic proliferation and atypical embryonic growth. Though almost all of complete hydatidiform moles are diploid androgenetic, scarce cases are biparental and caused mainly by mutations in NLRP7 and KHDC3L genes. NLRP7 mutations are more common and were reported in around $50-80 \%$ of cases from diverse populations while KHDC3 mutations were only found in 5-10\% of cases. Case description: A healthy 40-year-old Egyptian woman was referred to the Clinic of Prenatal Diagnosis and Fetal Medicine Department for counseling. She was married for 20 years to a first-degree relative and experienced 17 consecutive pregnancy losses without having any live births. Uterus ultrasound revealed endometrial thickening and subseptate uterus and in her last pregnancy failure, she complained of abdominal pain and severe shortness of breath. Immunochemistry tests were positive for $\beta$-human chorionic gonadotropin and histopathology-confirmed choriocarcinoma. Genetic testing revealed two novel heterozygous variants in the NLPR7 gene.

Conclusion: We presented a case with 17 recurrent hydatidiform moles that was complicated by choriocarcinoma due to novel variants in the NLRP7 gene.

Clinical significance: This is the first Egyptian case with recurrent hydatidiform mole. We identified novel NLPR7 variants, thus expanding the mutational spectrum associated with this rare disease.

Keywords: Choriocarcinoma, Egyptian patient, NLRP7 gene, Novel variants, Recurrent hydatidiform moles.

International Journal of Infertility and Fetal Medicine (2019): 10.5005/jp-journals-10016-1191
\end{abstract}

\section{BACKGROUND}

Hydatidiform mole (HM) is defined as an atypical human pregnancy characterized by the absence of abnormal embryonic growth and development with hydropic degeneration of chorionic villi. It is usually not recurrent and occurs once in every 1,000-1,500 pregnancies in Western countries. However, a higher incidence (up to a tenfold increase) was noted among underdeveloped and developing countries. ' In women with sporadic moles, $1-6 \%$ of cases encounter a second mole $\mathrm{e}^{2,3}$ and around $10-20 \%$ go through a second nonmolar reproductive loss, commonly known as sudden spontaneous abortion. ${ }^{4,5}$ Concerning recurrent HM (RHM), the frequency is not yet documented but such cases are assumed to be very rare. ${ }^{6}$

Hydatidiform mole is divided into two types according to the histopathological level: complete HM (CHM) or partial (PHM) based on the degree of trophoblast proliferation and the presence or absence of embryonic tissues beside the chorionic villi. Regarding the karyotype and genotype levels, most of the sporadic CHMs are diploid androgenetic, but may fall into these genotypes: diploid biparental, tetraploid androgenetic or biparental, aneuploid, triploid diandric, or even mosaic with two different cellular populations. ${ }^{7,8}$ Among the androgenetic moles, the commonest are monospermic while $5-20 \%$ are dispermic. In addition, almost all characterized recurrent HMs from women with no family history of moles were diploid and biparental with only two reported cases where one mole in a patient and three in another were shown to be diploid androgenetic. ${ }^{9}$

\begin{abstract}
1,3 Prenatal and Fetal Medicine Department, Human Genetics and Genome Research Division, National Research Centre, Cairo, Giza, Egypt

${ }^{2}$ Medical Molecular Genetics Department, Human Genetics and Genome Research Division, National Research Centre, Cairo, Giza, Egypt

Corresponding Author: Mai M Shaker, Prenatal and Fetal Medicine Department, Human Genetics and Genome Research Division, National Research Centre, Cairo, Giza, Egypt, Phone: +20 1005640802, e-mail: maishaker777@gmail.com

How to cite this article: Shalabi TA, Abdel-Hamid MS, Shaker MM. Two Novel Variants in NLRP7 Gene in an Egyptian Female Patient with Consecutive Molar Pregnancies Complicated by Choriocarcinoma. Int J Infertil Fetal Med 2019;10(3):54-57.
\end{abstract}

Source of support: Nil

Conflict of interest: None

Recurrent HM is caused mainly by biallelic mutations in two maternal-effect genes, NLRP7 and KHDC $3 L .^{10,11}$ The two genes encode proteins that play a pivotal role in maintaining the oocytes' integrity, cell-lineage differentiation, and postzygotic divisions. ${ }^{12}$ NLRP7 mutations are more common and were reported in around $50-80 \%$ of cases while $K H D C 3$ mutations were only found in $5-10 \%$ of cases. In about $10-20 \%$ of cases, no mutations were detected in the two genes suggesting the involvement of other unidentifiable genes. ${ }^{13}$

(-) The Author(s). 2019 Open Access This article is distributed under the terms of the Creative Commons Attribution 4.0International License (https://creativecommons. org/licenses/by-nc/4.0/), which permits unrestricted use, distribution, and non-commercial reproduction in any medium, provided you give appropriate credit to the original author(s) and the source, provide a link to the Creative Commons license, and indicate if changes were made. The Creative Commons Public Domain Dedication waiver (http://creativecommons.org/publicdomain/zero/1.0/) applies to the data made available in this article, unless otherwise stated. 
Herein, we describe an Egyptian female with 17 consecutive molar pregnancies, which were found to harbor two novel variants in the NLRP7 gene.

\section{Case Description}

A healthy 40-year-old Egyptian woman was referred to the outpatient clinic of Prenatal Diagnosis and Fetal Medicine Department, National Research Centre (NRC), for counseling. She had been married for 20 years to a first-degree relative and experienced 17 consecutive pregnancy losses with no live births. After each pregnancy loss, dilation and curettage was done and sample was taken for histopathology and results were consistent with complete vesicular mole. On examination, the patient had normal BMI and her blood pressure, glucose, and thyroid function tests were all normal. She was RH negative and the indirect Coombs test was done and showed negative results after each pregnancy loss. Cytogenetic studies were also carried out for the couple and revealed normal karyotype.

Uterus ultrasound revealed endometrial thickening and subseptate uterus. In the last pregnancy failure, the patient complained of abdominal pain and severe shortness of breath. Diagnostic workup was done and there was elevated chorionic gonadotropin levels of 2,820 $\mathrm{mlU} / \mathrm{mL}$. Immunochemistry tests were positive for $\beta$-human chorionic gonadotropin. In addition, histopathology revealed choriocarcinoma. The patient went on seven cycles of the chemotherapy regimen and was cured thereafter.

\section{Materials and Methods}

Genomic DNA was extracted from peripheral blood lymphocytes after having a signed informed consent according to the guidelines of the Medical Research Ethics Committee of the NRC, in accordance with Declaration of Helsinki. DNA was extracted using a standard extraction procedure. The coding regions and exon/intron boundaries of the NLRP7 and KHDC3L genes were amplified using specific primers designed by the Exon Primer software. Primers are available upon request from the corresponding author. Our standard PCR cycling conditions were: initial denaturation at $96^{\circ} \mathrm{C}$ for 5 minutes; 30 cycles of denaturation at $96^{\circ} \mathrm{C}$ for 30 seconds; annealing at $62^{\circ} \mathrm{C}$ for 30 seconds; extension at $72^{\circ} \mathrm{C}$ for 30 minutes, and a final extension at $72^{\circ} \mathrm{C}$ for 5 minutes. The PCR products were purified using Exo-SAP PCR Clean-up Kit (Fermentas, Germany) and sequenced in both directions using the BigDye Terminator v3.1 Cycle Sequencing Kit (Applied Biosystems, Foster City, CA, USA) and analyzed on the ABI Prism 3500 Genetic Analyzer (Applied Biosystems) according to manufacturer's instructions. The sequence data were compared with the reference genomic and CDNA sequence of the two genes, NLRP7 (NM_001127255) and KHDC3L (NM_001017361).

\section{Results}

The mutational analysis of the two genes revealed two novel heterozygous variants in the NLRP7 gene while no pathogenic variants were detected in the $K H D C 3 L$ gene. The two variants were one missense in exon 4 (c.1358T>G, p.lle453Ser) and one frameshift in exon 7 (c.2655dupC, p.lle886HisfsTer11) (Fig. 1). The c.2655dupC (p.lle886HisfsTer11) was maternally inherited while the c.1358T>G (p.lle453Ser) variant was not found in the mother's DNA and mostly inherited from the deceased father. The two variants were not found in the public genetic databases and were predicted to be causing disease by various bioinformatic tools. According to the American College of Medical Classification (ACMG) variants classification, the c.2655dupC (p.lle886HisfsTer11) was classified as "likely pathogenic" while the c.1358T>G (p.lle453Ser) was classified as "variant of uncertain significance."

\section{Discussion}

The NLRP7 is a nucleotide oligomerization domain (NOD)-like receptor, pyrin containing 7 , which maps to $19 q 13.4$ and was initially recognized as the causative gene for RHMs. Studies from several groups and populations agreed that NLRP7 is the main gene for this disorder and is mutated in $48-80 \%$ of females with a previous history of at least two HMs, reliant on patients' ascertainment criteria and populations. NLRP7 mutations differ in their way of inheritance from other monogenetic syndromes as females with NLRP7 mutations are healthy but they are at a high threat of reproductive failure. ${ }^{10,14}$ In this report, we described a new case with recurrent HM from Egypt (Fig. 2).

Normally, females older than 35 years have a twofold increased risk of molar pregnancy while those over 40 years have as high as five to tenfold increased risk. ${ }^{15}$ Nevertheless, a single study reported that recurrence of the disease (second molar pregnancy) appears to be present within 2-4 years after the first occurrence. Females should be followed up for a year if partial mole was diagnosed and for two years in case of a complete mole. ${ }^{16}$ The genetic background of HM is an important material for further investigation in many studies. NLRP7 gene mutations were found in familial forms and were repeated in many case reports. ${ }^{11,17}$ Scientists found that NLRP7 mutations were the cause for an increase in stochastic and mosaic aneuploidies during early stages of embryo development. There is a theory stating that the correlation between mutations of the NLRP7 gene and the formation of molar pregnancy is enlightened through the inability to express inflammatory response to many antigens and stimuli. ${ }^{6}$

The precise role of NLRP7 in the pathology of molar pregnancies is not fully clear, but existing data relate its protein to be involved in the inflammatory response with the trophoblastic lineage differentiation. ${ }^{18-20}$ Protein-truncating mutations, which are predictable to have major functional consequences on the protein, are associated with the absenteeism of embryonic tissues in the conceptions of these female patients. In contrast, missense mutations are usually associated with slighter functional consequences on the protein and the differentiation of certain embryonic tissues. ${ }^{21}$

Our case experienced 17 times complete vesicular mole without having any live births. She came from a rural area in Egypt where not having a child represents a social stigma. Therefore, she continuously tried to have a baby although was given several medical advices and counseling about the difficulty of having an offspring.

The mutational analysis of $N L R P 7$ and $K H D C 3 L$ genes revealed two variants in the NLRP7 gene. The two variants were one missense (c.1358T>G) in exon 4 and one frameshift (c.2655dupC) in exon 7. To our knowledge, these two variants were not described before in any case with HD. The c.1358T $>\mathrm{G}$ is a missense variant that results from the substitution of isoleucine by serine at position 453 (p.lle453Ser). On the other hand, the c.2655dupC involves the duplication of one nucleotide in exon 7 and is predicted to result in early protein truncation (p.lle886HisfsTer11). The two variants cosegregated with 


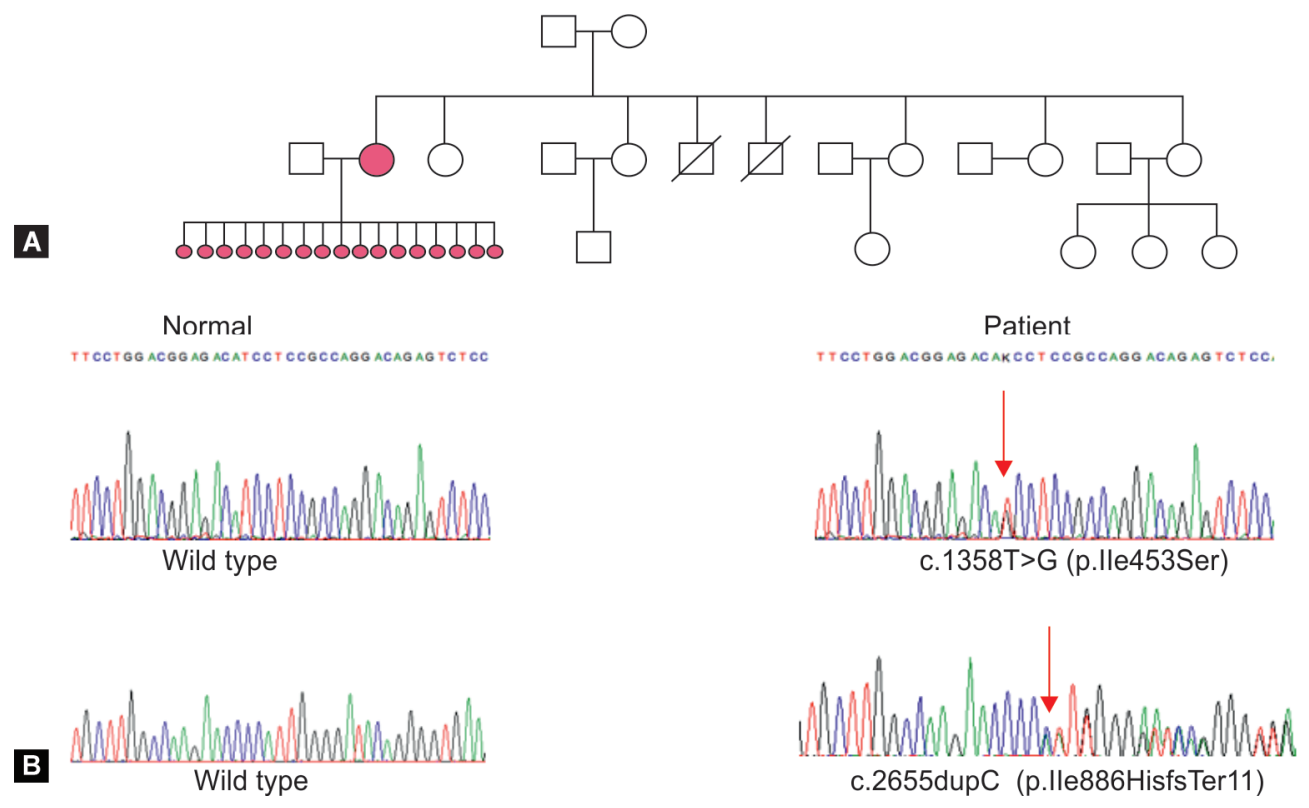

Figs $1 \mathrm{~A}$ and B: (A) Pedigree of the family; (B) Portion of the sequencing electrophoregram showing the two novel variants in the NLRP7 gene identified in our patient. Arrow indicates site of variant
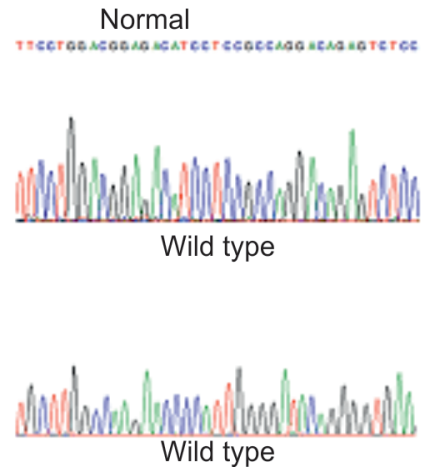
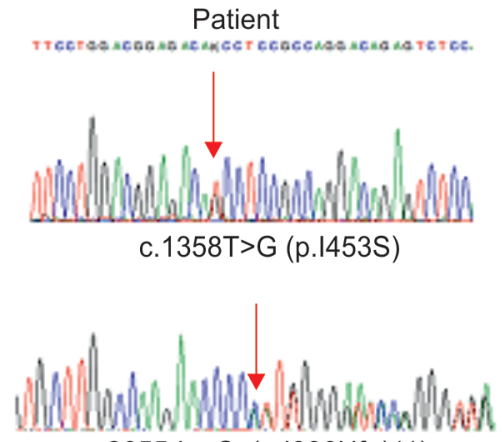
c.2655dupC (p.1886Hfs*11)

Fig. 2: DNA sequencing for two mutations; 1 missense in exon 4 (c.1358T>G, p.l453S) and 1 frameshift in exon 7 (c.2655dupC, p.l886Hfs*11)

the phenotype in our case as the frameshift variant was inherited from her mother while the missense variant was not found in the mother's DNA and we assumed that it was paternally inherited due to the lack of DNA from the deceased father.

To date, more than 60 distinct pathogenic variants have been reported in the NLRP7 gene. ${ }^{22-25}$ Majority of these mutations (>60\%) were protein truncating (nonsense, frameshift, or splice site) followed by missense mutations that were found in around $30-35 \%$ of cases. ${ }^{14,23-25}$ The c.2655dupC (p.lle886HisfsTer11) variant found in our case is located in the C-terminal LRR domain of the gene, which harbors majority of reported mutation. In contrast, the missense variant found in our patient (c.1358T > G, p.lle453Ser) is located in the NACHT domain of the gene. About 17 missenses have also been perceived as single heterozygous mutations or variants in females with recurrent moles. Though, the pathological consequence of these single mutations or variants is yet a topic of debate, and more data are needed to find a conclusion on their possible participation in the causation or genetic vulnerability for moles. ${ }^{13}$

Although NLRP7 and KHDC $3 L$ are the two main causative genes of $\mathrm{HMs}$, several cases were reported without having mutations in any of the two genes suggesting further genetic heterogeneity of the disorder. In this regard, Nguyen and coauthors performed whole exome sequencing for 65 women who were tested negative for both NLRP7 and KHDC3L. The study identified biallelic pathogenic variants in three new genes (MEI1, C11orf80, and REC114) in five unrelated women, two of them had other family members who experienced recurrent miscarriages and infertility. ${ }^{26}$

Counseling and management of females with NLRP7 mutations, egg donation, or preimplantation genetic diagnosis (PGD) have been proposed as a sole option for having a successful pregnancy. Within oocyte, the NLRP7 protein is transcribed from the maternal genome and is essential for regular fetal growth till the embryonic genome is activated. ${ }^{12}$ In latest studies, donor eggs having intact ooplasm lead to successful pregnancies in women with maternal effect variants in NLRP7. ${ }^{27}$ Though aberrant NLRP7 protein may be found in the maternal oocyte and ooplasm, PGD is focused on variants in the embryonic genome. Therefore, egg donation and PGD are possible initiators for successful pregnancy. Yet, more trials are necessary before accurate conclusions can be drawn. ${ }^{27}$

\section{Conclusion}

We presented an Egyptian case with recurrent HM that was complicated by choriocarcinoma. What is interesting in our case is the number of molar pregnancies the patient has had, in addition to the identification of two novel variants in the NLRP7 gene. 


\section{Clinical Significance}

This case report expands the mutational spectrum associated with this rare disease. Further, our results reinforce that NLRP7 gene mutations are the most common etiology of HD irrespective of ethnic variability.

All authors agree on publishing this work. A signed informed consent according to the guidelines of the Medical Research Ethics Committee of the NRC was obtained prior to sampling.

\section{References}

1. Grimes DA. Epidemiology of gestational trophoblastic disease. Am J Obstet Gynecol 1984;150(3):309-318. DOI: 10.1016/S00029378(84)90370-3.

2. Sebire NJ, Fisher RA, Foskett M, et al. Risk of recurrent hydatidiform mole and subsequent pregnancy outcome following complete or partial hydatidiform molar pregnancy. BJOG 2003;110(1):22-26. DOI: 10.1046/j.1471-0528.2003.02388.x.

3. Berkowitz RS, Im SS, Bernstein MR, et al. Gestational trophoblastic disease. Subsequent pregnancy outcome, including repeat molar pregnancy. J Reprod Med 1998;43(1):81-86.

4. Kim JH, Park DC, Bae SN, et al. Subsequent reproductive experience after treatment for gestational trophoblastic disease. Gynecol Oncol 1998;71(1):108-112. DOI: 10.1006/gyno.1998.5167.

5. Lan Z, Hongzhao S, Xiuyu Y, et al. Pregnancy outcomes of patients who conceived within 1 year after chemotherapy for gestational trophoblastic tumor: a clinical report of 22 patients. Gynecol Oncol 2001;83(1):146-148. DOI: 10.1006/gyno.2001.6170.

6. Deveault C, Qian JH, Chebaro W, et al. NLRP7 mutations in women with diploid androgenetic and triploid moles: a proposed mechanism for mole formation. Hum Mol Genet 2009;18(5):888-897. DOI: 10.1093/ $\mathrm{hmg} / \mathrm{ddn} 418$.

7. Vejerslev LO, Dissing J, Hansen HE, et al. Hydatidiform mole: Genetic markers in diploid abortuses with macroscopic villous enlargement. Cancer Genet Cytogenet 1987;26(1):143-155. DOI: 10.1016/01654608(87)90141-5.

8. Fukunaga M. Flow cytometric and clinicopathologic study of complete hydatidiform moles with special reference to the significance of cytometric aneuploidy. Gynecol Oncol 2001;81(1):6770. DOI: $10.1006 /$ gyno.2000.6100.

9. van der Smagt J, Scheenjes E, Kremer J, et al. Heterogeneity in the origin of recurrent complete hydatidiform moles: not all women with multiple molar pregnancies have biparental moles. BJOG 2006;113(6):725-728. DOI: 10.1111/j.1471-0528.2006.00929.x.

10. Murdoch S, Djuric U, Mazhar B, et al. Mutations in NALP7 cause recurrent hydatidiform moles and reproductive wastage in humans. Nat Genet 2006;38(3):300-302. DOI: 10.1038/ng1740.

11. Parry DA, Logan CV, Hayward BE, et al. Mutations causing familial biparental hydatidiform mole implicate C6orf221 as a possible regulator of genomic imprinting in the human oocyte. Am J Hum Genet 2011;89(3):451-458. DOI: 10.1016/j.ajhg.2011.08.002.

12. Akoury E, Li Z, Ao A, et al. NLRP7 and KHDC3L, the two maternal-effect proteins responsible for recurrent hydatidiform moles, co-localize to the oocyte cytoskeleton. Hum Reprod 2015;30(1):159-169. DOI: 10.1093/humrep/deu291.

13. Kalogiannidis I, Kalinderi K, Kalinderis M, et al. Recurrent complete hydatidiform mole: where we are, is there a safe gestational horizon? Opinion and mini-review. J Assist Reprod Genet 2018;35(6):967-973. DOI: 10.1007/s10815-018-1202-9.

14. Slim R, Wallace EP. NLRP7 and the genetics of hydatidiform moles: recent advances and new challenges. Front Immunol 2013;4:242. DOI: 10.3389/fimmu.2013.00242.

15. Palmer JR. Advances in the epidemiology of gestational trophoblastic disease. J Reprod Med 1994;39(3):155-162.

16. Lorigan PC, Sharma S, Bright N, et al. Characteristics of women with recurrent molar pregnancies. Gynecol Oncol 2000;78(3):288-292. DOI: 10.1006/gyno.2000.5871.

17. Wang $\mathrm{CM}$, Dixon $\mathrm{PH}$, Decordova $\mathrm{S}$, et al. Identification of 13 novel NLRP7 mutations in 20 families with recurrent hydatidiform mole; missense mutations cluster in the leucinerich region. J Med Genet 2009;46(8):569-575. DOI: 10.1136/jmg.2008.064196.

18. Khare S, Dorfleutner A, Bryan NB, et al. An NLRP7-containing inflammasome mediates recognition of microbial lipopeptides in human macrophages. Immunity 2012;36(3):464-476. DOI: 10.1016/j. immuni.2012.02.001.

19. Messaed C, Chebaro W, Roberto RBD, et al. NLRP7 in the spectrum of reproductive wastage: rare non-synonymous variants confer genetic susceptibility to recurrent reproductive wastage. Med Genet 2011;48(8):540-548. DOI: 10.1136/jmg.2011.089144.

20. Mahadevan S, Wen S, Wan YW, et al. NLRP7 affects trophoblast lineage differentiation, binds to overexpressed $Y Y 1$ and alters CpG methylation. Hum Mol Genet 2014;23(3):706-716. DOI: 10.1093/hmg/ ddt457.

21. Minh N, Nguyen P, Slim R. Genetics and epigenetics of recurrent hydatidiform moles: basic science and genetic counselling. Curr Obstet Gynecol Rep 2014;3(1):55-64. DOI: 10.1007/s13669-013-0076-1.

22. Reddy R, Nguyen NMP, Sarrabay G, et al. The genomic architecture of NLRP7 is Alu rich and predisposes to disease-associated large deletions. Eur J Hum Genet 2016;24(10):1445-1452. DOI: 10.1038/ ejhg.2016.9.

23. Soellner L, Begemann M, Degenhardt F, et al. Maternal heterozygous NLRP7 variant results in recurrent reproductive failure and imprinting disturbances in the offspring. Eur J Hum Genet 2017;25(8):924-929. DOI: 10.1038/ejhg.2017.94.

24. Fallahi J, Anvar Z, Razban V, et al. Founder effect of KHDC3L, p.M1V mutation, on iranian patients with recurrent hydatidiform moles. Iran J Med Sci 2020;45(2):118-124.

25. Allias F, Mechtouf N, Gaillot-Durand L, et al. A novel NLRP7 proteintruncating mutation associated with discordant and divergent p57 immunostaining in diploid biparental and triploid digynic moles. Virchows Arch 2020;477(2):309-315. DOI: 10.1007/s00428-02002769-w.

26. Nguyen NMP, Ge ZJ, Reddy R, et al. Causative mutations and mechanism of androgenetic hydatidiform moles. Am J Hum Genet 2018;103(5):740-751. DOI: 10.1016/j.ajhg.2018.10.007.

27. Akoury E, Gupta N, Bagga R, et al. Live births women with recurrent hydatidiform mole and two NLRP7 mutations. Reprod Biomed Online 2015;31(1):120-124. DOI: 10.1016/j.rbmo.2015.03.011. 